\title{
Analysis of mean and fluctuating helicity measured by To- moPIV in swirling jet
}

\author{
Rodion Stepanov ${ }^{1, \star}$, Peter Frick ${ }^{1}$, Vladimir Dulin ${ }^{2,3}$, and Dmitriy Markovich ${ }^{2,3}$ \\ ${ }^{1}$ Institute of Continuous Media Mechanics, Korolyov 1, Perm, 614013, Russia \\ ${ }^{2}$ Kutateladze Institute of Thermophysics, Lavrentyev av. 1, 630090 Novosibirsk, Russia \\ ${ }^{3}$ Novosibirsk State University, Pirogova 2, 630090 Novosibirsk, Russia
}

\begin{abstract}
Important role of helicity was theoretically predicted for the generation of large-scale magnetic fields and atmospheric vortices. Helicity can lead to a reduction of turbulent dissipation in the atmosphere or in a specific constrained flow, e.g. in pipe. We use the TomoPIV data (42 cube of grid points, resolution 0.84 $\mathrm{mm}$ ) to measure 3D velocity field of turbulent swirling flows. We study spatial distribution of the mean and fluctuating components of energy and helicity. We find that helical turbulence excitation and decay along stream of the jet strongly depend on the inflow swirl. We observe spatial separation of turbulent flow with different sign of helicity while integrated values are conserves. It is shown that large scale swirling flow induces helicity at the small scales. Our results bring valuable materials for benchmark the modern numerical simulations with turbulent closure technique.
\end{abstract}

\section{Introduction}

Helicity is a pseudoscalar characteristic of the flow which is defined by the correlation of the velocity and vorticity fields. It is known to be a topological characteristic of linkage of vortex lines [1]. In absence of viscosity the helicity is a conserved quantity as well as energy. Important role of helicity was theoretically predicted for the generation of large-scale magnetic fields (MHD dynamo) [2] and atmospheric vortices (hydrodynamic alpha effect) [3]. Helical features of atmospheric flows can be important to understated the tropical cyclone genesis $[4,5]$. Helicity can lead to a change of turbulent transport coefficients, e.g. reduction of turbulent dissipation [6, 7]. Different helical effects were revealed by theoretical studies or numerical simulations of 3D turbulence under idealized conditions: inverse energy cascade by helical triads [8], sustainment of large-scale vorticity field in a rotating turbulence with nonuniform helicity [9], reduction of direct energy cascade in highly helical turbulent flow $[10,11]$. However a comparison of experimental and theoretical results of helical phenomena remains a challenging problem.

Analysis of helicity generation, advection and dissipation mechanisms seems as an affordable task in frame of numerical simulations [12]. Experimental investigation of helicity of the mean flow is also possible. Mean velocity components can be measured with accuracy, enabling their differentiation to obtain the vorticity. However experimental study of helicity fluctuations in space and time is extremely difficult since they require measurements of

\footnotetext{
^e-mail: rodion@icmm.ru
}

instantaneous distributions of both velocity and vorticity vector fields. Helicity in MHD turbulence can be detected indirectly by measurements of magnetic field induction [13]. As far as we know there is only one successful attempt to measure the helicity power spectrum [14]. Modern optical techniques such as dual-plane or tomographic PIV (particle image velocimetry) make possible the reconstruction of helicity density distribution in a plane [15] or even in a volume [16]. The reconnection of linked vortex loops through helicity transformation demonstrated in recent successful experiments with high-speed scanning tomography apparatus [17]. In this work we use data of the tomographic PIV measurements of 3D velocity field in turbulent swirling jet for analysis of distribution of mean and fluctuating helicity. We suggest a processing of measurements that allow us to study the relation between the mean and fluctuating components of helicity and compare it with theoretical predictions.

\section{Experimental setup and flow configuration}

Swirling jets are generated in a transparent box by a convergent axisymmetric nozzle with mounted inside vane swirler (highly lighted part in figure 1). The detailed description of the experiment can be found in [16], where coherent structures in low-swirl (LS) and high-swirl (HS) turbulent jets are discussed. The Reynolds number $\mathrm{Re}=$ $U_{b} d / v$ is equal to 8900 (here $U_{b}=0.48 \mathrm{~m} / \mathrm{s}$ is the bulk velocity of the jet, $d=15 \mathrm{~mm}$ is the outlet diameter of the nozzle and $v$ is the viscosity). $3 \mathrm{D}$ velocity measure- 

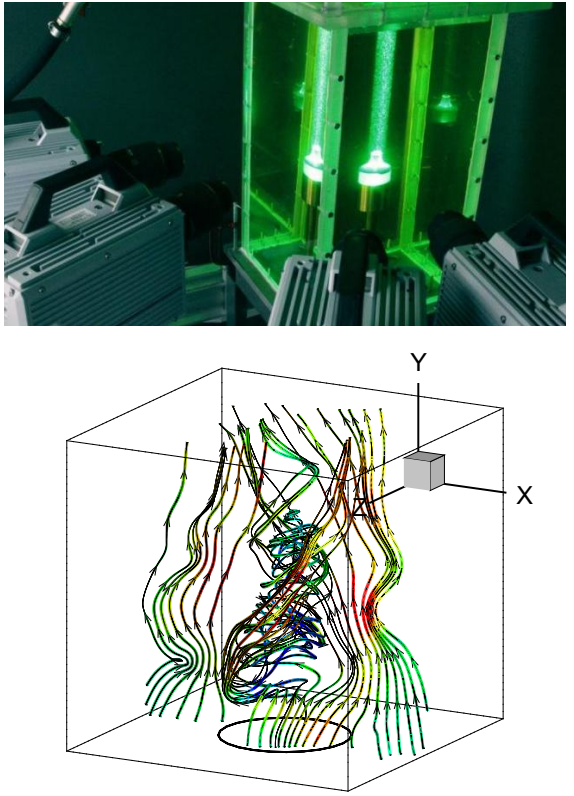

Fig. 1. Experimental setup (top) and structure of the swirling jet flow (bottom).

ments by tomographic PIV are performed for a cubic domain $40 \times 40 \times 40 \mathrm{~mm}$ downstream the nozzle rim. 2000 snapshots of 3D velocity are captured within one second, whereas the characteristic timescale is approximately forty times smaller [18]. Flow precession period for the HS jet is fifteen times smaller than the measurements interval. Reliable agreement between the mean velocity and Reynolds stresses obtained during the current high-repetition-rate experiments and previous low-frequency measurements is reported in [19].

In both, LS and HS jets, the superimposed swirl promotes development of helical instability modes in the jet mixing layer. The swirl is characterized by the swirl rate $S$, defined by the ratio of the angular jet momentum to the axial jet momentum, multiplied by the nozzle radius. $S=0.47$ and $S=1.0$ for LS and HS, respectively. These swirl intensities are correspondingly well below and above the critical swirl rate $S_{c r}=0.6$ for the swirling jet's vortex breakdown and formation of the recirculation zone at the jet axis [20]. Thus, in the latter case the flow is featured by a pronounced bubble-type vortex breakdown with flow precession. Direct 3D PIV measurements confirm the scenario, in which the flow dynamics is dominated by a global azimuthal instability mode, corresponding to a rotating coherent structure composed of spiraling vortex core and outer large-scale helical vortex [21].

\section{Results}

Applying decomposition of total fields in the mean and fluctuating parts to the velocity $\mathbf{U}=\mathbf{V}+\mathbf{v}$ and vorticity $\nabla \times \mathbf{U}=\mathbf{W}+\mathbf{w}$ (by definition, $\langle\mathbf{U}\rangle=\mathbf{V},\langle\mathbf{v}\rangle=0$ and time averaging is supposed for statistically stationary flow), one gets the energy of the mean flow $E=|\mathbf{V}|^{2}$ and energy
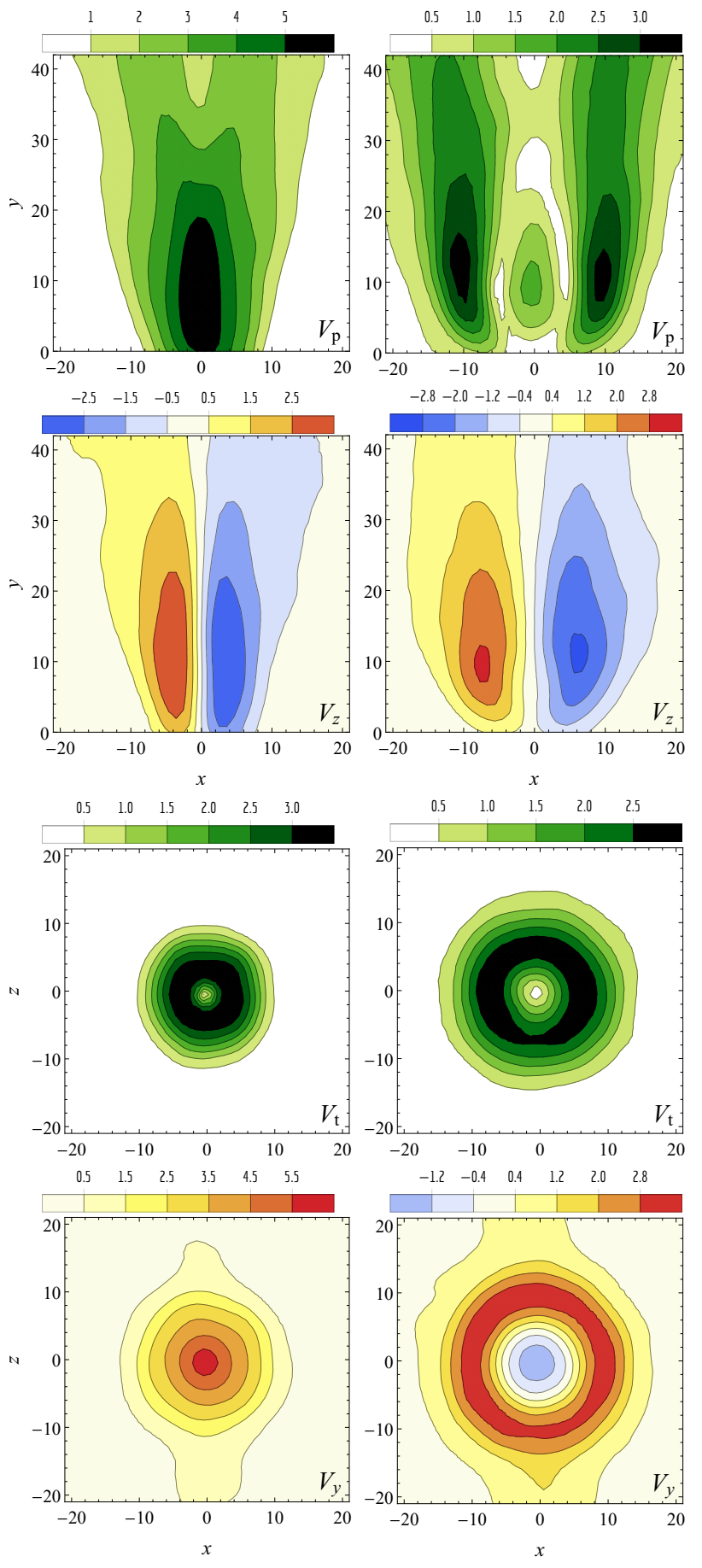

Fig. 2. Mean velocity components in the plane $z=0$ (two top rows) and in the plane $y=10$ (two bottom rows): LS (left column), HS (right column) jets.

of fluctuations $e=\left\langle|\mathbf{v}|^{2}\right\rangle$ and the helicity of mean flow $H=\mathbf{V} \cdot \mathbf{W}$ and helicity of fluctuations $h=\langle\mathbf{v} \cdot \mathbf{w}\rangle$. We analyze $E, e, H$ and $h$ separately to understand the relative dynamics of energy and helicity in the turbulent flow.

In figure 2 we show the distributions of velocity components in the central vertical cross-section $(z=0)$ and in one selected horizontal cross-section at $y=10$ of both LS and HS jets. Velocity components perpendicular to the presented plains are demonstrated by its values with sign ( 

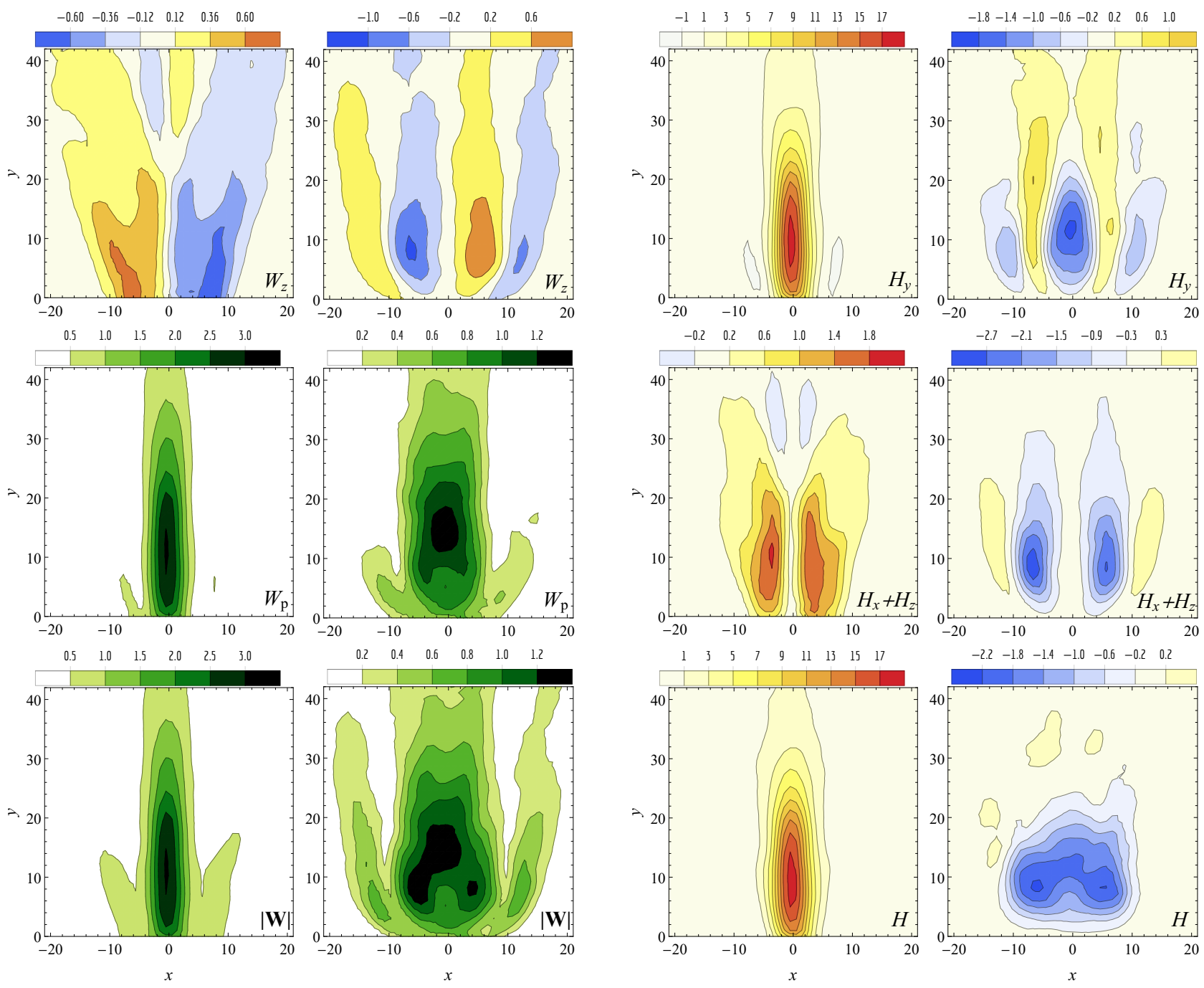

Fig. 3. Mean flow vorticity in the plane $z=0$ for LS (left column) and HS (right column) jets: $W_{z}$ (top row), intensity $W_{\mathrm{p}}$ (middle row), total intensity $|\mathbf{W}|$ (bottom row).

$V_{z}$ and $V_{y}$ ) while velocity components parallel to the presented plains are depicted by its absolute values ( intensity of poloidal velocity component $V_{\mathrm{p}}=\left(V_{x}^{2}+V_{y}^{2}\right)^{1 / 2}$ and intensity of toroidal velocity component $\left.V_{\mathrm{t}}=\left(V_{x}^{2}+V_{z}^{2}\right)^{1 / 2}\right)$. The horizontal cross-section at $y=10$ is selected because the HS jet has inner region with reversed flow at this hight. Dimensional quantities here and below are given in units of length $0.07 \mathrm{~mm}$ and units of time $0.0005 \mathrm{~s}$ so unit of velocity is $0.14 \mathrm{~m} / \mathrm{s}$.

The difference is more pronounced in the structure of mean vorticity $\mathbf{W}$ presented in figure 3 . In the LS jet the map of the $W_{z}$ component reveals a strong toroidal vortex nearby the nozzle and a weak contrarotating toroidal vortex at the downstream. In the HS jet this contrarotating vortex goes down to the nozzle pushing aside the weakened lower vortex. The distribution of total vorticity intensity becomes also more complicate in HS jet.

Resulting helicity of the mean flow is shown in figure 4. We split the total helicity in two parts: the parallel and perpendicular to jet flow, $H_{y}=V_{y} W_{y}$ and $H_{\mathrm{t}}=$

Fig. 4. Helicity of mean flow in the plane $z=0$ for LS (left column) and HS (right column) jets: $H_{y}$ (top row), $H_{x}+H_{z}$ (middle row) and $H$ (bottom row).

$V_{x} W_{x}+V_{z} W_{z}$. The structure of helicity field is completely different in two jets. In both jets a strong anisotropy is seen, the $H_{y}$ component dominates in LS jet, while $H_{\mathrm{t}}$ is stronger in HS jet. Moreover, the sign of dominating helicity is opposite in LS and HS. Thus, the vortex breakdown in HS jet and formation of the central recirculation zone locally change the sign of the mean flow helicity.

Characteristics of fluctuating fields are shown in figure 5 and confirm the particular difference between LS and HS flows. Note that the similar structure of energy and enstrophy distributions (correspondingly, $e$ and $e_{w}=\left\langle|\mathbf{w}|^{2}\right\rangle$ ) in each jet (LS and HS) mean a moderate isotropy of fluctuations. Sign defined helicity of fluctuations indicates a correlation of $\mathbf{v}$ and $\mathbf{w}$ fields (see figure 6). Again one can see strong difference in sign and distribution of small-scale helicity between LS and HS. The accordance of the dominating sign of $h$ and $H$ in each flow supports the transfer of helicity from large scales to small scales.

It is interesting to follow the evolution of helicity, mainly generated at the onset of jet flow, along the jet. We 

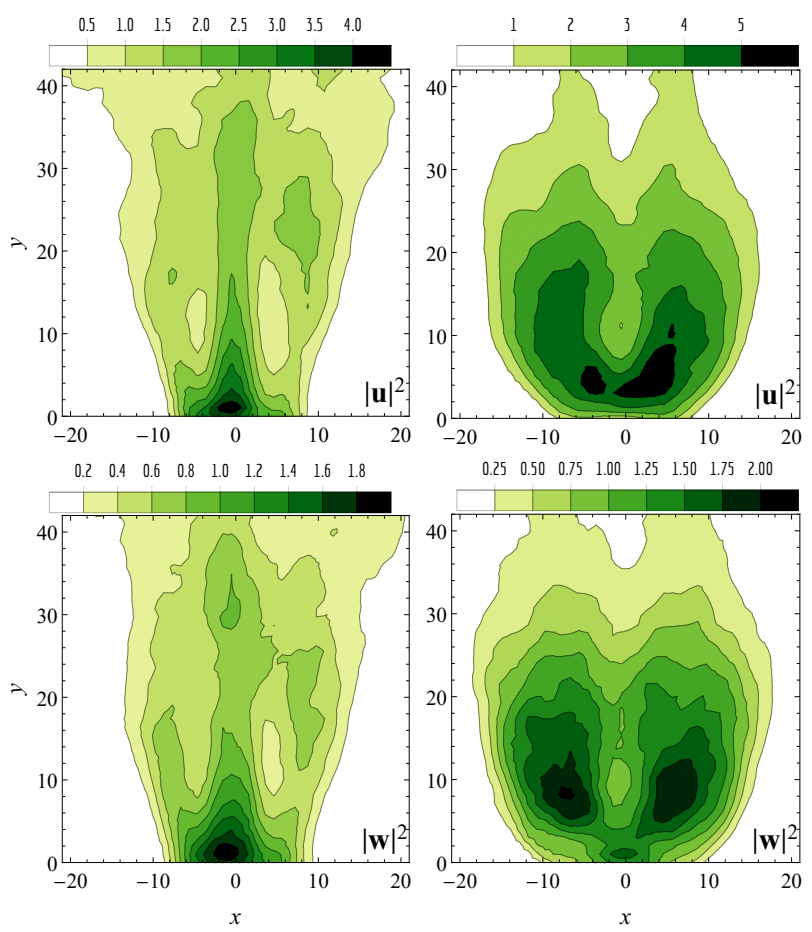

Fig. 5. Characteristics of fluctuations in the plane $z=0$ for LS (left column) and HS (right column) jets: energy of velocity fluctuations $e$ (top row), enstrophy of fluctuations $e_{w}=\left\langle|\mathbf{w}|^{2}\right\rangle$ (bottom row).

plot in figure 7 the $y$-dependency of flow characteristics integrated over $x O z$ plane, which illustrate the generation and decay of helical turbulence. The large-scale helicity is generated in the beginning of the jet up to $y \approx 10$. Note again that the helicity input rate depends on the degree of swirling in a very complicate way. Moderate swirling produce positive helicity, i.e. the sign of helicity corresponds to the hand of helix in the nozzle. The strong swirling increases the opening angle and generates an inverse axial flow, producing negative mean-flow (large-scale) helicity. In both jets the mean-flow helicity reaches the maximum at $y \approx 10$ (although HS evolves faster) and monotonically decays in what follows. In HS the decay is essentially faster - the mean-flow helicity vanishes at $y \approx 30$. The energy of the mean flow at $y>30$ becomes very similar in both jets. More surprisingly seams the equality of energy of fluctuations at large $y$, because at the maximum (at $y \approx 12$ ) the energy of fluctuations in HS was about 5 times larger as in LS. Note, that despite different spatial distributions of helicity components $H_{y}$ and $H_{\mathrm{t}}$ the integrated values are getting in balance. Similar character of evolution (isotropization) was found by a helicity analysis in the swirling pipe flow [22].

The mean helicity of fluctuations in LS jet is very weak at low $y$. It is slightly negative and changes the sign in the cross-section, where $H$ reaches the maximum. Downstream, the small-scale helicity monotonically increases up to $y \approx 30$. In this part of the jet $(10<y<30)$ the energy of small-scale pulsations also increases, supporting the concept of energy and helicity and helicity transfer

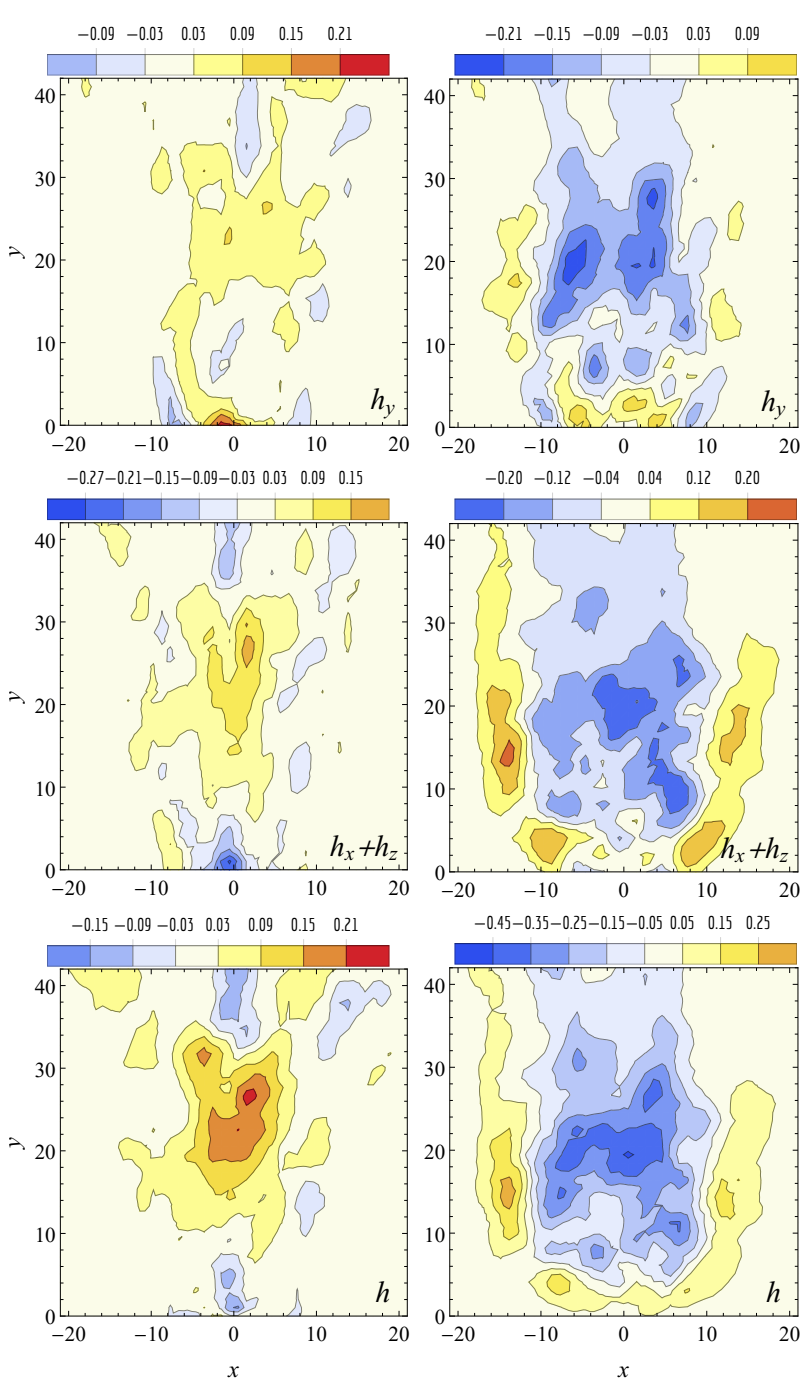

Fig. 6. Helicity of fluctuations in the plane $z=0$ for LS (left column) and HS (right column) jets: $h_{y}$ (top row), $h_{\mathrm{t}}=h_{x}+h_{z}$ (middle row) and total $h$ (bottom row).

from large scales to small scales. Helicity of fluctuations in two horizontal cross-sections at $y=16$ and $y=32$ are shown in figure 8.

\section{Conclusions}

Tomographic particle image velocimetry measurements of turbulent swirling jets allows us to study the mean and pulsation components of the flow. Spatial distribution of energy and helicity reveals the anisotropic structure of the jet flow. Scenario of helicity generation and decay along the stream of the jet depends dramatically on the inflow swirl. It may correspond to bifurcation character of filament formation of helical vortex which depends on swirl intensity $[16,21,23]$. We observe spatial separation of turbulent flow with different sign of helicity while integrated values are conserved. Applying time and space averaging we demonstrated energy and helicity transfers from large scales to small scales. Our results bring valuable materials to benchmark the modern numerical simulations with turbulent closure technique, i.e. LES. Smogarinski's model 

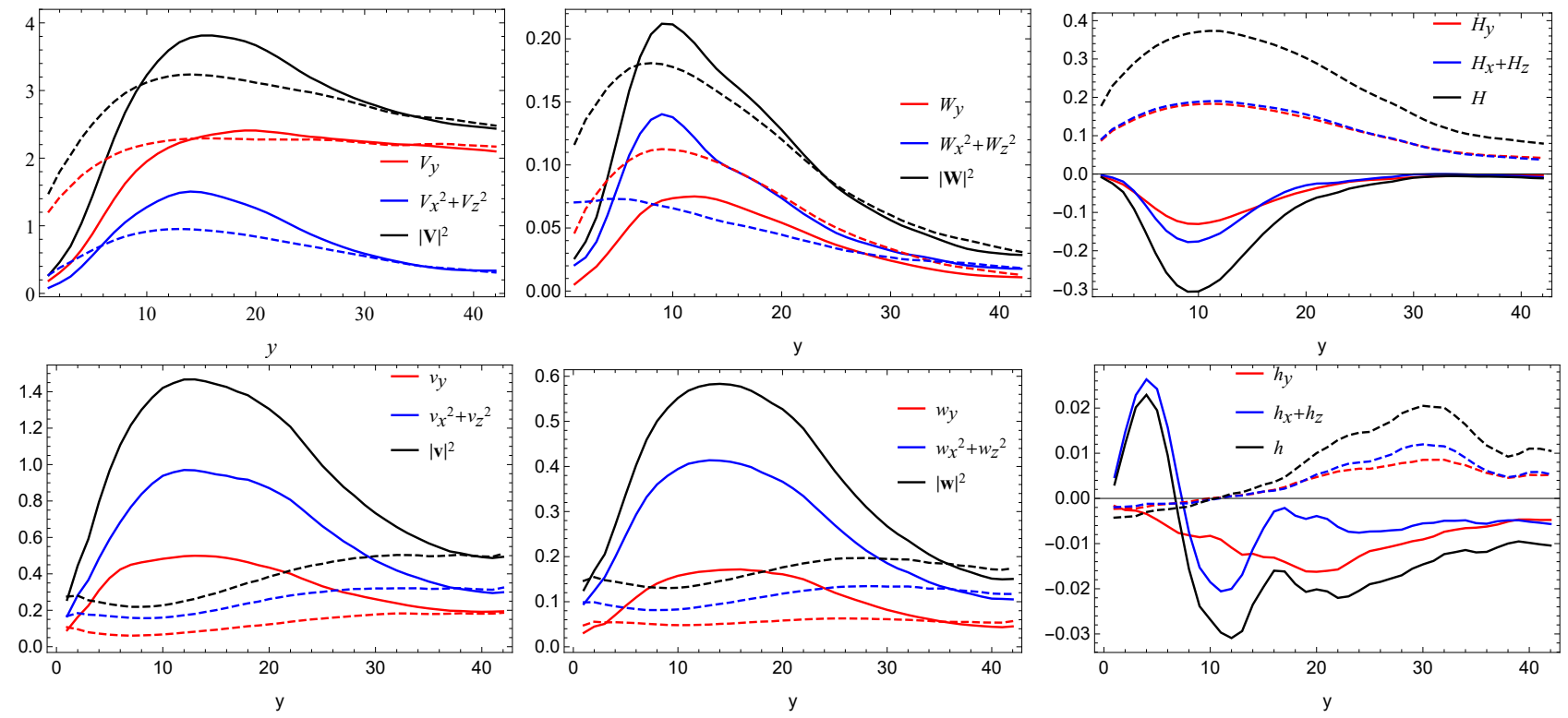

Fig. 7. Dependencies of flow characteristics integrated over $x O z$ plain on $y$ coordinate (along jet stream) for LS (dashed lines) and HS (solid lines) jets.
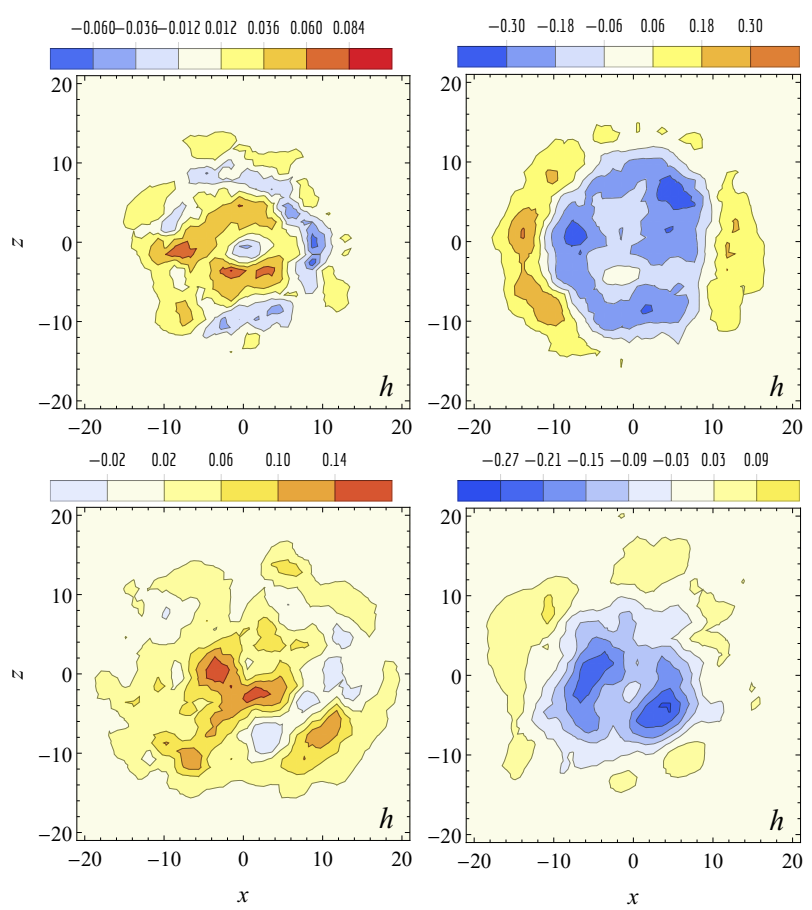

Fig. 8. Helicity of fluctuations in the planes $y=16$ (top row) and $y=32$ (bottom row) for LS (left column) and HS (right column) jets.

including helicity and inhomogeneity of turbulence might be revised and improved.

Acknowledgements. This work was supported by the Russian Science Foundation (grand RSF-16-41-02012).

\section{References}

[1] H.K. Moffatt, J. Fluid Mech. 35, 117-129 (1969)
[2] M. Steenbeck, F. Krause, K.H. Rädler, Z. Naturforsch., Part A 21, 369 (1966)

[3] S.S. Moiseev, R.Z. Sagdeev, A.V. Tur, G.A. Khomenko, A.M. Shukurov, Sov. Phys. Dokl. 28, 926 (1983)

[4] G. Levina, M. Montgomery, Doklady Earth Sciences 434, 1285 (2010)

[5] G.V. Levina, M.T. Montgomery, Doklady Earth Sciences 458, 1143 (2014)

[6] D.K. Lilly, J. Atmos. Sci. 43, 126 (1986)

[7] N. Yokoi, A. Yoshizawa, Phys. Fluids 5, 464 (1993)

[8] L. Biferale, S. Musacchio, F. Toschi, Phys. Rev. Lett. 108, 164501 (2012)

[9] N. Yokoi, A. Brandenburg, Phys. Rev. E 93, 033125 (2016), 1511.08983

[10] R. Stepanov, E. Golbraikh, P. Frick, A. Shestakov, Phys. Rev. Lett.115, 234501 (2015), 1508.07236

[11] M. Kessar, F. Plunian, R. Stepanov, G. Balarac, Phys. Rev. E 92, 031004 (2015)

[12] A. Teimurazov, A. Sukhanovskii, A. Evgrafova, R. Stepanov, Journal of Physics: Conference Series 899, 022017 (2017)

[13] R. Stepanov, R. Volk, S. Denisov, P. Frick, V. Noskov, J.F. Pinton, Phys. Rev. E73, 046310 (2006)

[14] B.M. Koprov, V.M. Koprov, V.M. Ponomarev, O.G. Chkhetiani, Dokl. Phys. 50, 419 (2005)

[15] G.S. Regunath, W.B. Zimmerman, V. Tesař, B.N. Hewakandamby, Exp. Fluids 45, 973 (2008)

[16] D.M. Markovich, V.M. Dulin, S.S. Abdurakipov, L.A. Kozinkin, M.P. Tokarev, K. Hanjalić, J. Turbulence 17, 678 (2016)

[17] D. Kleckner, W.T.M. Irvine, Nat. Phys. 9, 253 (2013) 
[18] S.V. Alekseenko, V.M. Dulin, Y.S. Kozorezov, D.M. Markovich, International Journal of Heat and Fluid Flow 29, 1699 (2008)

[19] V. Dulin, S. Abdurakipov, D. Markovich, K. Hanjalic, Dynamics of large-scale vortices in nonswirling and swirling turbulent jets. Time-resolved tomographic PIV measurements, in Proceedings of the 10th International Symposium on Turbulence and Shear Flow Phenomena, edited by L. Smits, A. Johansson, M. Oberlack, H.J. Sung, S. Tavoularis,
H. Nagib (2017), Vol. 3, p. 233

[20] H. Liang, T. Maxworthy, Journal of Fluid Mechanics 525, 115 (2005)

[21] K. Oberleithner, C. Paschereit, R. Seele, I. Wygnanski, AIAA journal 50, 1437 (2012)

[22] A. Vasiliev, A. Sukhanovskii, R. Stepanov, IOP Conference Series: Materials Science and Engineering 208 (2017)

[23] L.G. Sarasua, A.C. Sicardi Schifino, R. González, Physics of Fluids 17, 044104 (2005) 